\title{
Fukutin, identified by the Escherichia coli ampicillin secretion trap (CAST) method, participates in tumor progression in gastric cancer
}

\author{
Htoo Zarni Oo ${ }^{1}$ Kazuhiro Sentani ${ }^{1}$ Shoichiro Mukai $^{1} \cdot$ Takuya Hattori $^{1}$. \\ Shunsuke Shinmei ${ }^{1}$ - Keisuke Goto ${ }^{1}$ - Naoya Sakamoto ${ }^{1}$ - Yutaka Naito ${ }^{1}$. \\ Katsuhiro Anami ${ }^{1} \cdot$ Pharm Thi Binh Trang ${ }^{1} \cdot$ Kazuyoshi Yanagihara $^{2}$. \\ Naohide Oue ${ }^{1} \cdot$ Wataru Yasui $^{1}$
}

Received: 15 February 2015 / Accepted: 6 June 2015/Published online: 30 July 2015

(C) The International Gastric Cancer Association and The Japanese Gastric Cancer Association 2015

\begin{abstract}
Background Gastric cancer (GC) is the fifth commonest malignancy worldwide and still one of the leading causes of cancer-related death. The aim of this study was to identify a novel prognostic marker or therapeutic target for GC.

Methods We analyzed candidate genes from our previous Escherichia coli ampicillin secretion trap (CAST) libraries in detail, and focused on the FKTN gene because it was overexpressed in both GC cell line CAST libraries, MKN-1 and MKN-45.

Results Quantitative reverse transcriptase PCR analysis of $F K T N$ revealed that $F K T N$ messenger RNA was overexpressed in nine of $28(32.1 \%)$ GC tissue samples compared with nonneoplastic gastric mucosa. Immunostaining of fukutin showed that 297 of 695 cases $(42.7 \%)$ were positive for fukutin. Fukutin-positive GC cases were significantly associated with differentiated histological features, and advanced $\mathrm{T}$ grade and $\mathrm{N}$ grade. In addition, fukutin expression was observed more frequently in the intestinal phenotype $(51 \%)$ of GC than in other phenotypes $(37 \%)$ when defined by the expression patterns of
\end{abstract}

Electronic supplementary material The online version of this article (doi:10.1007/s10120-015-0511-2) contains supplementary material, which is available to authorized users.

Wataru Yasui

wyasui@hiroshima-u.ac.jp

1 Department of Molecular Pathology, Hiroshima University Institute of Biomedical and Health Sciences, 1-2-3 Kasumi, Minami-ku, Hiroshima 734-8551, Japan

2 Division of Translational Research, Exploratory Oncology and Clinical Trial Center, National Cancer Center Hospital East, 6-5-1 Kashiwanoha, Kashiwa, Chiba 277-8577, Japan mucin 5AC, mucin 6, mucin 2, and CD10. FKTN small interfering RNA treatment decreased GC cell proliferation. Conclusions These results indicate that the expression of fukutin may be a key regulator for progression of GC with the intestinal mucin phenotype.

Keywords Gastric cancer - FKTN $\cdot$ Fukutin $\cdot$ Cell growth $\cdot$ Mucin phenotype

\section{Introduction}

Gastric cancer (GC) remains a major public health problem worldwide, and is the fifth commonest human malignant disease. Although therapeutic outcomes have recently improved for early GC, it remains one of the world's leading causes of cancer-related death [1]. Generally, GCs have been classified into two histological types on the basis of the tendency toward gland formation-namely, intestinal type and diffuse type according to Lauren [2], or differentiated type and undifferentiated type according to Nakamura et al. [3]. GCs are also classified as having a gastric phenotype, a gastric and intestinal mixed phenotype, or an intestinal phenotype depending on the expression of mucin phenotypic markers [4-6]. The mucin expression and phenotype of tumors are associated with clinicopathological findings and tumorigenesis in GCs. However, the clinical importance of intestinal mucin in GCs is still controversial and, at the time of writing, no definite conclusions have been reached [5, 6].

Better knowledge of the changes in gene expression that occur during gastric carcinogenesis may lead to improvements in diagnosis, treatment, and prevention. Comprehensive gene expression analysis is useful to identify potential molecular markers for $\mathrm{GC}$ and to better 
understand the development of GC at the molecular level. We previously performed several large-scale gene expression studies using array-based hybridization [7] and serial analysis of gene expression [8,9], and identified a number of genes, including REG4 (which encodes regenerating islet-derived protein 4) [10,11] OLFM4 (which encodes olfactomedin 4) [12], BPIFAl, also known as PLUNC (which encodes palate, lung, and nasal epithelium carcinoma associated protein) [13], and GJB6 (which encodes connexin 30) [14]. Our recent study of Escherichia coli ampicillin secretion trap (CAST) analysis on GC cell lines and cancer tissues identified several candidate genes. Desmocollin 2 (DSC2) expression was associated with GC of the intestinal mucin phenotype with CDX2 expression [15] and transmembrane 9 superfamily member 3 (TM9SF3) expression was significantly associated with tumor progression and involved in cancer cell invasion [16]. These results indicated the methods to be useful for the identification of novel genes associated with GC; however, such alterations cannot completely explain the pathogenesis of GC.

In the present study, we analyzed candidate genes from previous CAST libraries in detail, and focused on the FKTN gene (which encodes fukutin) because it was overexpressed in both GC cell line CAST libraries, MKN-1 and MKN-45. Quantitative reverse transcriptase PCR (RTPCR) analysis showed the expression of FKTN to be relatively higher in GC tissue than in corresponding nonneoplastic gastric mucosa. FKTN is responsible for Fukuyama-type congenital muscular dystrophy. Fukutin is presumably involved in the glycosylation of $\alpha$-dystroglycan, which is involved in basement membrane formation. Moreover, FKTN has been reported as a potential tumor suppressor gene in human cervical and breast cancer cell lines [17]. To our knowledge, however, the detailed function and expression profiles of the FKTN gene in human GC have not yet been elucidated.

This is the first detailed analysis of fukutin expression in human GC. Here, we clarify the upregulation of FKTN messenger ENA (mRNA) expression via quantitative RTPCR analysis of surgically resected GC tissues. To clarify the pattern of expression and localization of fukutin in GC and association with clinocopathological characteristics, we performed immunohistochemical analysis of surgically resected GC samples and analyzed the association between fukutin and various markers, including the gastric and intestinal mucin phenotypes (mucin-5AC, mucin 6, mucin 2 and CD10), and CDX2. Furthermore, using a small interfering (siRNA) knockdown system, we examined the biological role of $F K T N$ in cancer cell proliferation and invasion in GC cell lines.

\section{Materials and methods}

\section{Tissue samples}

Fresh tissue samples of primary tumor and the corresponding nonneoplastic gastric mucosa were collected from 28 patients with GC who received surgical treatment at Hiroshima University Hospital or affiliated hospitals. For RNA and protein extraction, tissue samples obtained at the time of surgery were immediately embedded in O.C.T. compound (Sakura Finetechnical, Tokyo, Japan), frozen in liquid nitrogen, and stored at $-80{ }^{\circ} \mathrm{C}$. Samples of normal brain, spinal cord, heart, skeletal muscle, lung, stomach, small intestine, colon, liver, pancreas, kidney, uterus, bone marrow, spleen, peripheral leukocytes, and trachea were purchased from Clontech (Palo Alto, CA, USA). For immunohistochemical analysis, we used archival formalinfixed paraffin-embedded tissues from 695 patients who had undergone surgical excision of GC at Hiroshima University Hospital. The 695 GC cases were histologically classified as differentiated type $(n=359)$ and undifferentiated type $(n=336)$, according to the Japanese classification of gastric carcinoma [18]. Tumor staging was done according to the TNM classification. Because written informed consent was not obtained, identifying information for all samples was removed before analysis for strict privacy protection. This procedure was in accordance with the ethical guidelines for human genome/gene research enacted by the Japanese government.

\section{Quantitative RT-PCR and Western blot}

Quantitative RT-PCR was performed with an ABI PRISM 7900 sequence detection system (Applied Biosystems, Foster City, CA, USA) as described previously [19]. We calculated the ratio of target gene mRNA expression levels between GC tissue $(T)$ and corresponding nonneoplastic mucosa $(N) . T / N$ ratios greater than 2 were considered to represent overexpression. $\beta$-Actin (ACTB gene) was used as a housekeeping internal control. Western blot was performed as described previously [20].

\section{Antibodies}

Anti-fukutin antibody (ab131280) was purchased from Abcam (Tokyo, Japan). Four antibodies were used for the analysis of the GC phenotypes: anti-mucin 5AC (Novocastra, Newcastle, UK) as a marker of gastric foveolar epithelial cells, anti-mucin 6 (Novocastra) as a marker of pyloric gland cells, anti-mucin 2 (Novocastra) as a marker of goblet cells in the small intestine and colorectum, and 
anti-CD10 (Novocastra) as a marker of microvilli of absorptive cells in the small intestine and colorectum. AntiCDX2 (BioGenex, San Ramon, CA, USA) was used as a marker of differentiation of intestinal epithelial cells.

\section{Immunohistochemistry}

An LSAB kit (Dako, Carpinteria, CA, USA) was used for immunohistochemical analysis. In brief, sections were pretreated by microwave irradiation in citrate buffer for $15 \mathrm{~min}$ to retrieve antigenicity. Then peroxidase activity was blocked with $3 \%$ hydrogen peroxide-methanol for $10 \mathrm{~min}$. Sections were incubated with the following primary antibodies: anti-fukutin (1:200 dilution, anti-rabbit monoclonal antibody) and anti-mucin 5AC, anti-mucin 6, anti-mucin 2, and anti-CD10 (all diluted 1:50, anti-mouse monoclonal antibodies). Sections were incubated with primary antibody for $1 \mathrm{~h}$ at $25^{\circ} \mathrm{C}$, followed by incubations with biotinylated secondary IgG and peroxidase-labeled streptavidin for 10 min each. Staining was completed with 10 min incubation with substrate-chromogen solution. The sections were counterstained with $0.1 \%$ hematoxylin. For fukutin expression, in specimens with more than $10 \%$ staining of cancer cells, the immunostaining was considered positive according to median cutoff values rounded off to the nearest $5 \%$ (range $0-80$ ). GC cases were classified into four phenotypes as described previously: gastric phenotype, intestinal phenotype, gastric and intestinal mixed phenotype, and unclassified phenotype [21].

\section{RNA interference}

RNA interference was performed to knock down endogenous FKTN. Small interfering RNA (siRNA) oligonucleotides for $F K T N$ and a negative control were purchased from Invitrogen (Carlsbad, CA, USA). Three independent oligonucleotides were used for FKTN siRNA as follows: FKTN siRNA-1 sequence, 5'-GCA ACC CAA UGG AAU CUG GCC UAU U-3'; FKTN siRNA-2 sequence, 5'-CCA CUU GAG ACU UAA AGA ACA CAU U-3'; and FKTN siRNA-3 sequence, 5'-GGC CUU UCG GAA GAG UGC AAA GGA A-3'. Transfection was performed using Lipofectamine RNAiMAX reagent (Invitrogen) according to the manufacturer's protocol. Briefly, 100 pmol siRNA and $12 \mu \mathrm{L}$ Lipofectamine RNAiMAX were mixed in $1 \mathrm{~mL}$ RPMI medium (10 nmol/L final siRNA concentration). After 20 min of incubation, the mixture was added to the cells and plated on tissue culture dishes. The analyzed cells were incubated for $48 \mathrm{~h}$ after transfection in all experiments.
Cell lines, cell growth, and in vitro invasion assays

Eight cell lines derived from human GC were used. GC cell lines of the MKN series-MKN-1 (adenosquamous cell carcinoma), MKN-7 and MKN-74 (well-differentiated adenocarcinoma), and MKN-45 (poorly differentiated adenocarcinoma)-were purchased from the Japanese Collection of Research Bioresources Cell Bank (Osaka, Japan). KATO-III, HSC-39 (signet ring cell carcinoma), and HSC57 (well-differentiated adenocarcinoma) were provided by Morimasa Sekiguchi (University of Tokyo) [22]. HSC44PE (signet ring cell carcinoma with scirrhous variant) was provided by Kazuyoshi Yanagihara [23]. The TMK-1 cell line was established in our laboratory from a poorly differentiated adenocarcinoma [24]. All cell lines were maintained in RPMI 1640 (Nissui Pharmaceutical, Tokyo, Japan) containing $10 \%$ fetal bovine serum (BioWhittaker, Walkersville, MD, USA) in a humidified atmosphere of $5 \% \mathrm{CO}_{2}$ and $95 \%$ air at $37{ }^{\circ} \mathrm{C}$. The MKN-1 and MKN-45 cells were seeded at a density of 2000 cells per well in 96-well plates. Cell growth was monitored after day 0, 1, 2, and 4 for the 3-(4,5-dimethylthiazol-2-yl)-2,5-diphenyltetrazolium bromide (MTT) assay, as described elsewhere [25]. Modified Boyden chamber assays were performed to examine invasiveness. Cells were plated at 200,000 cells per well in RPMI-1640 medium plus $1 \%$ serum in the upper chamber of a Transwell insert $(8-\mu \mathrm{m}$ pore diameter; Chemicon, Temecula, CA, USA) coated with Matrigel. Medium containing $10 \%$ serum was added in the bottom chamber with use of a 24-well plate format. On days 1 and 2 , noninvading cells in the upper chamber were removed by a clean cotton swab, and the cells attached to the lower surface of the insert were stained with Cell Stain (Chemicon, Temecula, CA, USA). The invading cells were then counted with an ordinary light microscope.

\section{Statistical methods}

Correlations between clinicopathological parameters and fukutin staining were analyzed by the chi squared test. $P<0.05$ was considered statistically significant. Statistical analyses were performed with JMP (version 9.0.2; SAS Institute, Carey, NC, USA). Another gene expression profile and survival data were available on a total of $265 \mathrm{GC}$ patients from The Cancer Genome Atlas (TCGA) website (http://www.cbioportal.org/) [26]. The Kaplan-Meier method was used to estimate the overall survival, and the difference in survival among groups was examined with a log-rank test. 


\section{Results}

\section{FKTN overexpression in GC cell line CAST libraries}

We previously generated CAST libraries from two GC cell line CAST libraries-MKN-1 [15], and MKN-45 [16]and normal stomach [15]. CAST is a useful method for identifying novel biomarkers and new therapeutic targets. One hundred fifty-two genes and 237 genes were found in the known databases that were specifically expressed in GC cell line MKN-1 and MKN-45 CAST libraries, respectively, and this means that these genes were not found in the normal stomach CAST library. In the present study, we selected only genes that were detected at least two times in each GC cell line CAST library but not in the normal stomach CAST library. Furthermore, we selected only genes that have not been investigated in GC. FKTN was the top candidate expressed in both CAST libraries (Table S1).

\section{Messenger RNA expression and protein expression of FKTN in systemic normal organs, GC cell lines, and GC tissues}

Genes expressed at high levels in tumors and very low levels in normal tissues are ideal diagnostic markers and therapeutic targets. To confirm whether the FKTN gene is cancer specific, quantitative RT-PCR was performed in nine GC tissue samples and in 14 kinds of normal tissue (liver, kidney, heart, colon, brain, bone marrow, skeletal muscle, lung, small intestine, spleen, spinal cord, stomach, pancreas, and peripheral leukocyte). FKTN expression was detected at low levels or to a lesser extent in normal organs, including the stomach. High FKTN expression was observed in three of nine GC tissues ((33.3\%; Fig. 1a). To validate the CAST data, FKTN expression in GC was investigated by quantitative RT-PCR in an additional 28 GC samples and corresponding nonneoplastic mucosa. We calculated the ratio of target gene mRNA expression levels between GC tissue $(T)$ and corresponding nonneoplastic mucosa $(N) . T / N$ ratios greater than 2 were considered to represent overexpression. FKTN mRNA was overexpressed in nine of 28 cases (32.1\%; Fig. 1b). We next examined the FKTN expression in the TCGA Stomach Adenocarcinoma RNA sequencing database [26], and we found that 109 of 265 GC cases (41\%) had FKTN mRNA upregulation. Western blot analysis showed that fukutin was observed in all eight GC cell lines, irrespective of the histological subtypes. In addition, the fukutin levels were upregulated in three GC tissues compared with corresponding noncancerous tissues (Fig. 3a).
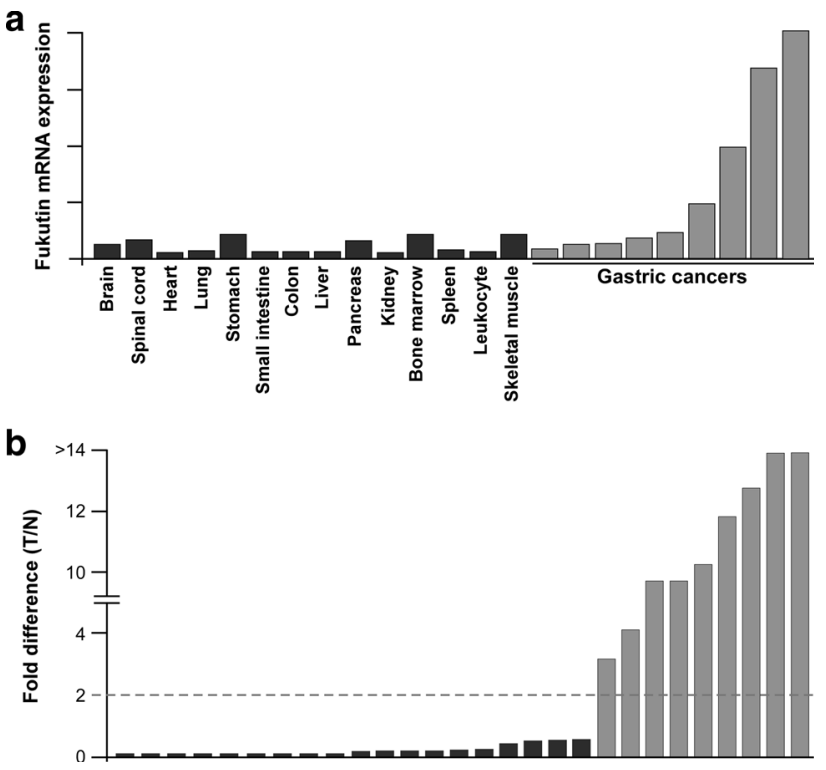

Fig. 1 Quantitative reverse transcriptase PCR analysis of FKTN. a FKTN messenger RNA (mRNA) expression level in 14 normal tissues and nine gastric cancer (GC) samples (arbitrary units). b $T$ / $N$ ratio of FKTN mRNA level between GC tissue $(T)$ and corresponding nonneoplastic mucosa $(N)$ in $28 \mathrm{GC}$ cases. A $T / N$ ratio greater than 2 was considered to represent overexpression. Upregulation of the FKTN gene was observed in $32 \%$ of the cases

\section{Immunohistochemical analysis of fukutin in GC}

To analyze the tissue localization, pattern of distribution, and the relationship between clinicopathological parameters and fukutin in GC, immunohistochemical analysis of fukutin was performed with a commercially available antibody. Fukutin expression was detected in 297 of 695 GCs $(42.7 \%)$, and it showed cytoplasmic granular staining in cancer cells, from the superficial layer to the deep layer, especially in differentiated-type GC (Fig. 2a, b). In nonneoplastic gastric mucosa, weak staining of fukutin was observed in intestinal metaplastic cells (Fig. 2f), but not in the normal gastric epithelial glands. Next, the relationship between fukutin expression and clinicopathological parameters was examined. Fukutin-positive expression showed a significant correlation with the depth of invasion $(P=0.0125)$ and lymph node metastasis $(P=0.0104)$. There was no significant association between fukutin expression and the other parameters (stage or $\mathrm{M}$ grade). Histologically, fukutin was observed more frequently in differentiated-type GC than in undifferentiated-type GC $(P<0.0001)$ (Table 1$)$. The role of fukutin in survival prognosis of $93 \mathrm{GC}$ patients was examined, but no significant correlation between fukutin expression and overall survival was found (data not shown). Furthermore, the 
Fig. 2 Immunohistochemical analysis in GC tissues and nonneoplastic gastric mucosa. a Fukutin expression in differentiated-type GC tissue. b Fukutin was observed as cytoplasmic staining in cancer cells. c-e Intestinal gastric mucin phenotypes were examined: CD10 and mucin 2 (MUC2). Serial section showed that CD10 expression was partly adjacent to cytoplasmic expression of fukutin. f Fukutin expression was detected in intestinal metaplastic cells but not in the normal stomach mucosa. g Expression of fukutin was observed more frequently in intestinal-type GC than in the other GC mucin phenotypes. $P$ values were statistically analyzed by Fisher's exact test
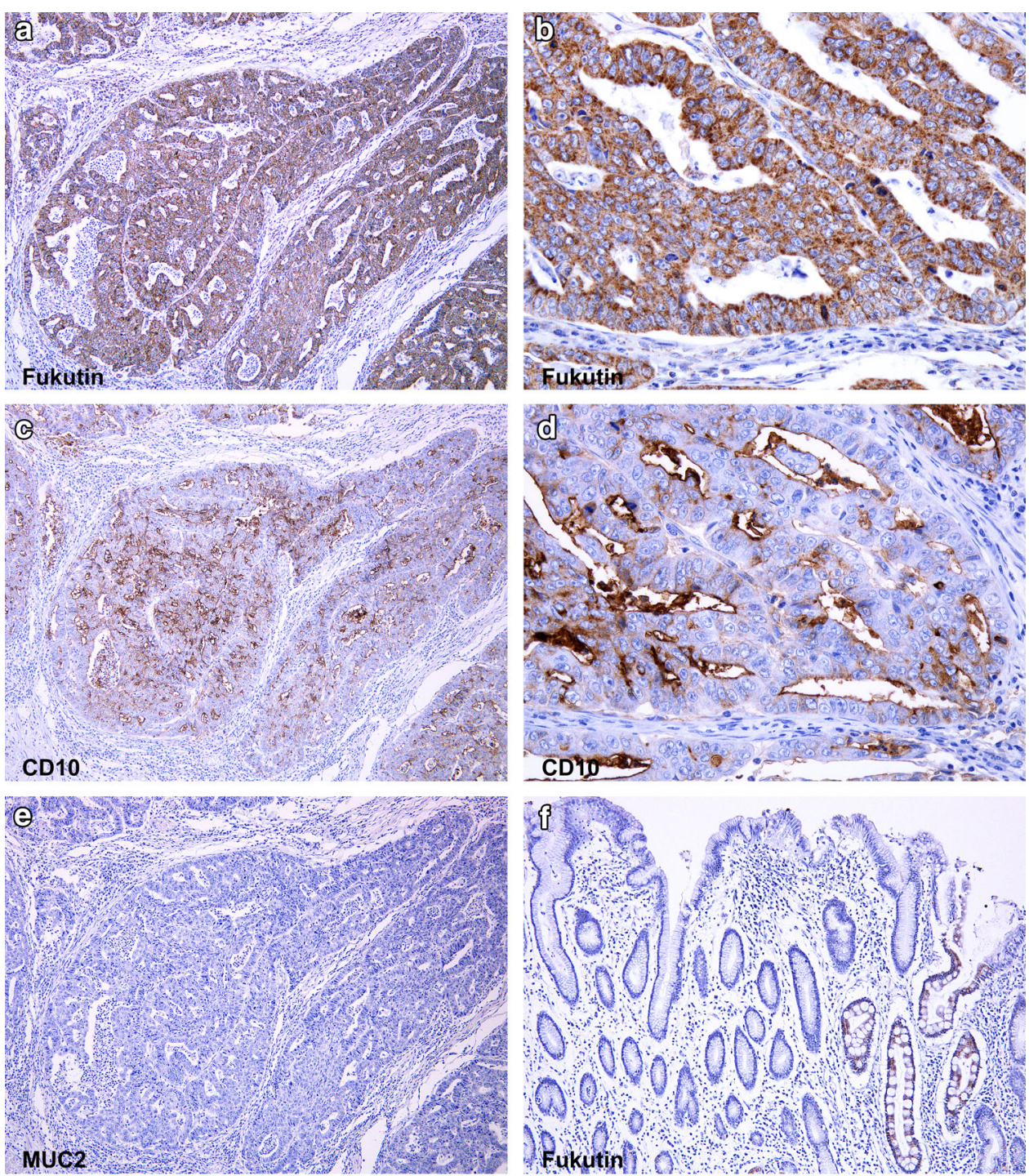

g

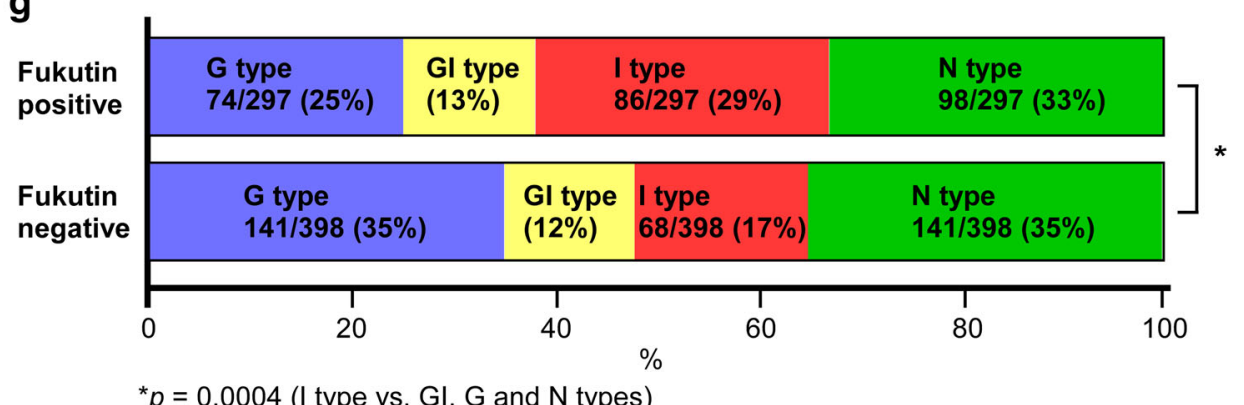

RNA sequencing data from TCGA provided the survival analysis between GC cases with and without FKTN mRNA upregulation [26]. However, there was no statistical significant correlation between survival prognosis and FKTN mRNA expression status (Fig. S1).

\section{Association between gastric or intestinal mucin markers and fukutin expression}

We next investigated the association between fukutin expression and various markers determining the gastric and 
Table 1 Relationship between FKTN expression and clinicopathological parameters in 695 gastric cancer (GC) cases obtained by immunohistochemistry

\begin{tabular}{lccc}
\hline & \multicolumn{2}{l}{$F K T N$ expression } & \multirow{2}{*}{$P$} \\
\cline { 2 - 3 } & Positive & Negative & \\
\hline Age & & & \\
$\quad \leq 65$ years $(n=336)$ & $130(39 \%)$ & 206 & 0.037 \\
$>65$ years $(n=359)$ & $167(47 \%)$ & 192 & \\
Gender & & & \\
Female $(n=261)$ & $93(36 \%)$ & 168 & 0.0032 \\
Male $(n=434)$ & $204(47 \%)$ & 230 & \\
T grade & & & \\
T1 $(n=198)$ & $70(35 \%)$ & 128 & 0.0125 \\
T2/T3/T4 $(n=497)$ & $227(45 \%)$ & 270 & \\
N grade & & & \\
N0 $(n=296)$ & $110(37 \%)$ & 186 & 0.0104 \\
N1/N2/N3 $(n=399)$ & $187(46 \%)$ & 212 & \\
M grade & & & \\
M0 $(n=680)$ & $287(42 \%)$ & 393 & NS \\
M1 $(n=15)$ & $10(66 \%)$ & 5 & \\
Stage & & & \\
Stage I $(n=284)$ & $114(40 \%)$ & 170 & NS \\
Stage II/III/IV $(n=411)$ & $183(44 \%)$ & 228 & \\
Histology & & & \\
Differentiated $(n=359)$ & $236(60 \%)$ & 123 & \\
Undifferentiated $(n=336)$ & $61(18 \%)$ & 275 & \\
\hline
\end{tabular}

$P$ values were calculated by Fisher's exact test.

NS not significant

intestinal phenotypes. In the 695 cases examined, each molecule was detected in 274 cases $(39.4 \%)$ for mucin 5AC, 95 cases $(13.6 \%)$ for mucin 6,158 cases $(22.7 \%)$ for mucin 2, and 89 cases (12.8\%) for CD10. The $695 \mathrm{GC}$ cases were classified into four phenotypes: 215 (30.9\%) were of the gastric phenotype, $87(12.5 \%)$ were of the gastric and intestinal mixed phenotype, $154(22.1 \%)$ were of the intestinal phenotype, and $239(34.3 \%)$ were of the unclassified phenotype. The positive expression of fukutin was significantly more frequent in CD10-positive cases than in CD10-negative cases $(P<0.0001)$ (Table 2$)$. In immunohistochemical staining, the localized distribution of fukutin and CD10 was partly contiguous (Fig. 3c, d). However, there was no clear relationship between the expression of fukutin and other markers (mucin 5AC, mucin 6, and mucin 2) (Fig. 2e, Table 2). Fukutin expression occurred more frequently in the intestinal phenotype than in other phenotypes of GC $(P=0.0004)$ (Fig. 2f). The correlation between fukutin expression and intestinal differentiation marker CDX2 was also analyzed, but no significant relationship was found (data not shown).
Table 2 Relationship between $F K T N$ expression and gastric/intestinal mucin markers in $695 \mathrm{GC}$ cases

\begin{tabular}{llll}
\hline & \multicolumn{2}{l}{$F K T$ expression } & $P$ \\
\cline { 2 - 3 } & Positive & Negative & \\
\hline Mucin 5AC & & \\
Positive $(n=274)$ & $106(37 \%)$ & 168 & NS \\
Negative $(n=421)$ & $191(45 \%)$ & 230 & \\
Mucin 6 & & & \\
Positive $(n=95)$ & $42(44 \%)$ & 53 & NS \\
Negative $(n=600)$ & $255(43 \%)$ & 345 & \\
Mucin 2 & & & \\
Positive $(n=158)$ & $69(44 \%)$ & 89 & NS \\
Negative $(n=537)$ & $228(42 \%)$ & 309 & \\
CD10 & & & \\
Positive $(n=89)$ & $58(65 \%)$ & 31 & $<0.0001$ \\
Negative $(n=606)$ & $239(39 \%)$ & 367 & \\
\hline
\end{tabular}

$P$ values were calculated by Fisher's exact test.

Fig. 3 Effect of fukutin downregulation on cell growth and cell invasion. a The anti-fukutin antibody was detected in the band at approximately $53 \mathrm{kDa}$ on Western blot of eight GC cell lines. $\beta$-Actin was used as a loading control. The fukutin levels were upregulated in three GC tissues compared with corresponding noncancerous tissues. b Western blot analysis of FKTN in MKN-1 and MKN-45 GC cells transfected with negative control small interfering (siRNA) or FKTN siRNAs (siRNA-1, siRNA-2, siRNA-3). c Cell growth was assessed by a 3-(4,5-dimethylthiazol-2-yl)-2,5-diphenyltetrazolium bromide (MTT) assay on 96-well plates in MKN-1 and MKN-45 cells. d Effect of FKTN knockdown on cell invasion in MKN-1 and MKN-45 cells. MKN-1 and MKN-45 GC cells transfected with negative control siRNA or FKTN siRNA-1 and siRNA-3 were incubated in Boyden chambers. After 24 and $48 \mathrm{~h}$ of incubation, invading cells were counted. Bars and error bars show the mean and standard deviation, respectively, of three different experiments. Asterisk $P<0.05, O . D$ optical density, $N S$ not significant

\section{Effect of fukutin downregulation on cell proliferation and invasive activity}

To analyze the biological significance of fukutin in GC, siRNA knockdown was performed on the MKN-1 and MKN-45 GC cell lines and confirmed by Western blot analysis (Fig. 3a, b). To determine the possible role of fukutin in the proliferative effect of GC cells, an MTT assay was performed. Cell proliferative ability was significantly abrogated in FKTN knockdown GC cells compared with negative control siRNA-transfected GC cells (Fig. 3c). The possible invasiveness of fukutin was also investigated. A Transwell invasion assay was performed 2 days after negative control siRNA and FKTN siRNA transfection in MKN-1 and MKN-45 cells. There was no 
a

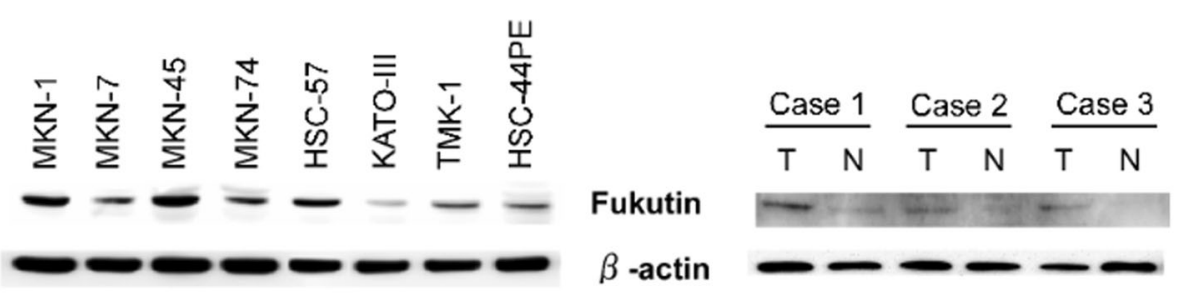

b

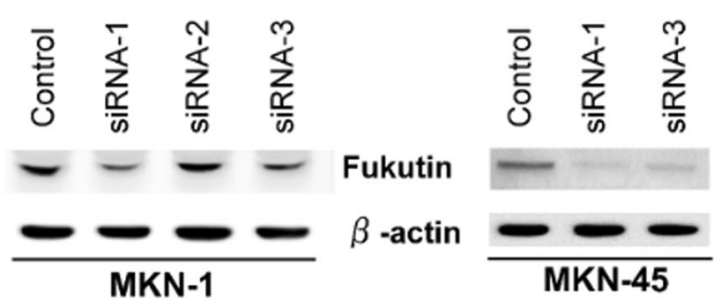

MTT assay

C

MKN-1
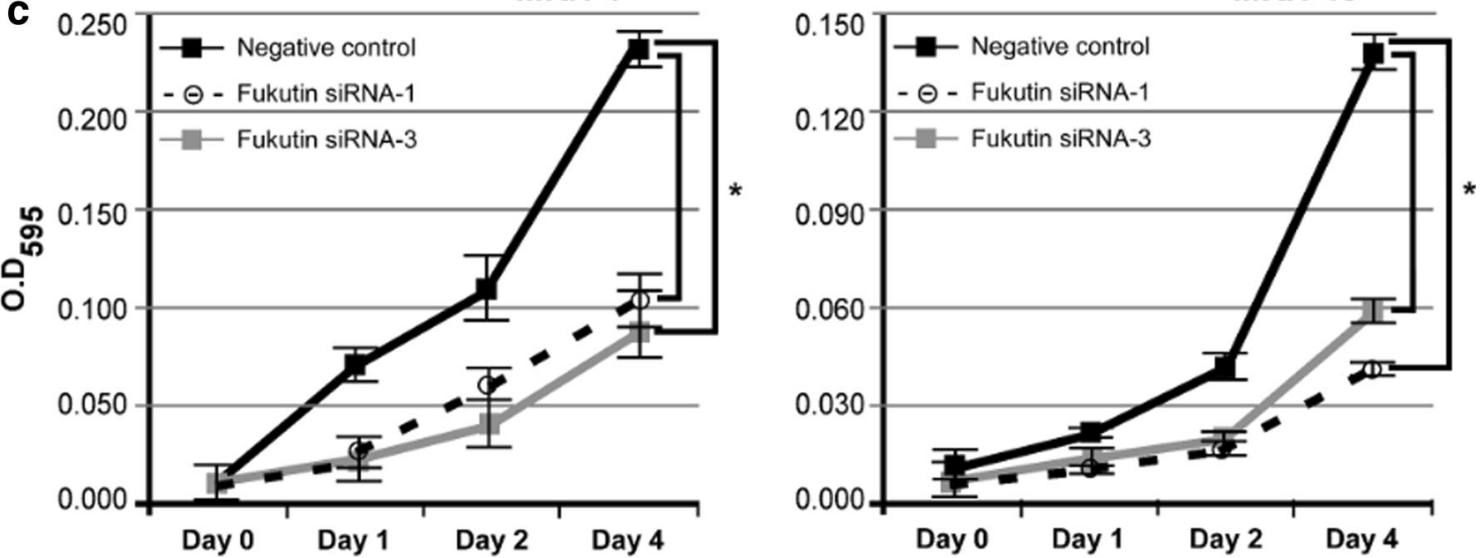

d

Invasion assay $\quad$ MKN-1
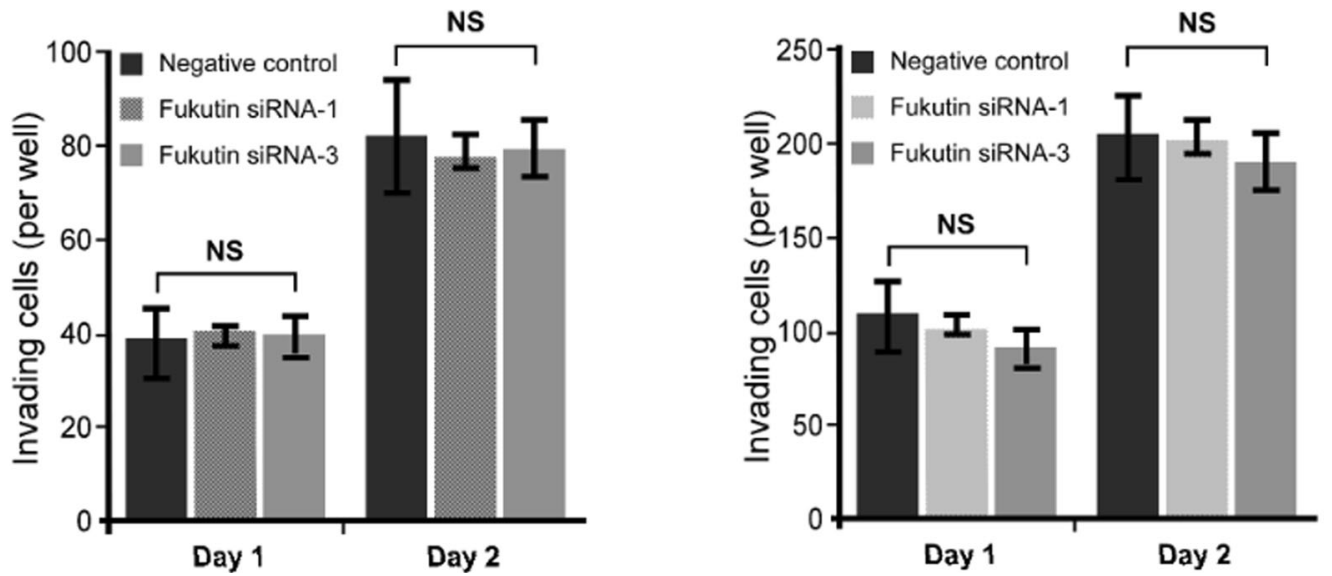
significant difference in the invasion rate between control and knockdown cells (Fig. 3d). To clarify the molecular signaling pathways associated with proliferation activities, we examined the phosphorylation of epidermal growth factor receptor (EGFR) downstream molecules as described previously [27]. FKTN knockdown did not affect the levels of EGFR, Akt, extracellular-signal-regulated kinase $1 / 2$, or p38 and their phosphorylated forms (data not shown).

\section{Discussion}

In this study, he gene expression profile was studied with GC cell lines that were previously analyzed by the CAST method. We focused on FKTN as a novel target gene among genes that were specifically upregulated in GC. The rationale for the in-depth analysis of FKTN was based on three main reasons: firstly, because of its frequent overexpression in two GC cell line CAST libraries, MKN-1 and MKN-45; secondly, quantitative RT-PCR analysis revealed that $F K T N$ was more frequently overexpressed in GC tissue than in nonneoplastic gastric mucosa; and thirdly, the expression and biological significance of FKTN in human GC have not been investigated. Here, we showed by the immunohistochemical analysis of 695 human GC cases that FKTN expression was significantly associated with differentiated-type GC and the expression of intestinal mucin phenotype marker CD10. Furthermore, knockdown of FKTN by RNA interference was found to inhibit cancer cell proliferation in GC cell lines. Taken together, these results suggest that fukutin constitutes a potential therapeutic target in GC.

$F K T N$, which encodes fukutin, is presumably related to the glycosylation of $\alpha$-dystroglycan, which is involved in basement membrane formation. Saito and Matsumura [28] showed a severe reduction in the binding activity of $\alpha$-dystroglycan for ligands, such as laminin, agrin, or neurexin, after $F K T N$ knockdown. It indicated that the loss of function of FKTN results in defective glycosylation of $\alpha$-dystroglycan, a central component of the dystrophinglycoprotein complex, leading to the disruption of the linkage between the basal lamina and the cytoskeleton. The binding of laminin to $\alpha$-dystroglycan causes signaling through the dystorglycan-syntrophin-Grb2-SOS1-Rac1PAK1-JNK cascade, which is initiated by Src family kinases and which also causes syntrophin tyrosine phosphorylation to begin signaling [29]. Disruption of the linkage between $\alpha$-dystroglycan and laminin is predicted to have profound effects on muscle-cell viability, because it causes destabilization of the sarcolemma against contraction-stretch stress, hampers signal transduction, and inhibits the assembly of extracellular matrix proteins. The downregulation of fukutin has been observed in Fukuyamatype congenital muscular dystrophy [30,31], which affects both sexes almost equally. FKTN expression is reported to influence female sexual maturation or age of menarche [32]. The present study showed a significant relationship between FKTN expression and age and gender, but the detailed mechanism in GC remains unclear.

Immunohistochemical analysis showed that 297 of 695 GC cases $(43 \%)$ displayed fukutin expression. Consistent with our data, FKTN mRNA expression is upregulated in $41 \%$ of GC cases in the TCGA Stomach Adenocarcinoma database [26], and this means both the protein and the transcript levels are upregulated in more than $40 \%$ of GCs. However, FKTN upregulation is not directly correlated with overall survival of GC patients, and it reflects that fukutin could be a diagnostic or therapeutic target but not a prognostic marker for GC. On the other hand, fukutin was scarcely expressed in normal gastric mucosa and surrounding nonneoplastic gastric mucosa. Golgi accumulation of fukutin is often observed in intestinal metaplasia as well as in GC of the intestinal phenotype, implying that these changes occur at an early stage of stomach carcinogenesis. Once carcinogenesis has started, fukutin might enhance tumor growth and signaling between extracellular matrix from glycosylation of dystroglycan, which would promote laminin and extracellular matrix interaction. With regard to the mucin phenotype, there was a significant correlation between fukutin expression and the expression of CD10. CD10 expression varied with tissue origin; in prostate cancer, $\mathrm{CD} 10$ expression was positively correlated with elevated prostate-specific antigen level, higher Gleason score, and advanced stage. Moreover, prostate-specific antigen recurrence was significantly associated with the staining pattern and subcellular localization of CD10 [33]. In contrast, in ovarian cancer, overexpression of CD10 enhanced susceptibility to paclitaxel, resulting in an increased occurrence of apoptosis, as well as downregulation of both matrix metalloproteinase 2 and vascular endothelial growth factor expression [34]. In colorectal cancer, CD10 expression is associated with colorectal cancer metastases, especially liver metastasis [35]. In GC, CD10 is more prominently expressed in well to moderately differentiated adenocarcinoma than in poorly differentiated GC [36], and stromal CD10 expression in differentiated GC strongly promoted invasion and metastasis [37].

At present, there are no studies in the literature concerning the biological function and role of fukutin in GC. Knockdown of FKTN resulted in decreased cell proliferation in comparison with negative control cells. In the present study, FKTN knockdown did not affect the levels of EGFR downstream molecules significantly. Yamamoto et al. [38] showed that immunoprecipitation and an ELISAbased RNA-binding assay demonstrated possible binding 
between FKTN mRNA and Musashi-1. The role of Musashi-1 in GC has been reported. It was shown that Musashi-1 expression is higher in intestinal-type GC and that it was significantly correlated with lymph node metastasis, but not with distant metastasis or the depth of invasion [39]. It is presumed that fukutin in GC might be regulated by Musashi-1 and might activate molecular signaling pathways other than the EGFR pathway, such as Wnt and Notch pathways.

In conclusion, the potential of FKTN for treating GC by gene therapy is promising. However, extensive study is required to elucidate the molecular mechanisms of its activity in tumor cell biology. Evaluating the molecular mechanism of fukutin involvement in tumor cell growth might improve our understanding of GC carcinogenesis and tumor progression.

Acknowledgments We thank Shinichi Norimura for his excellent technical assistance and advice. This work was performed with the kind cooperation of the Research Center for Molecular Medicine and Analysis Center of Life Sciences, Hiroshima University. This work was supported in part by Grants-in-Aid for Cancer Research from the Ministry of Education, Culture, Science, Sports and Technology of Japan, in part by a Grant-in-Aid for the Third Comprehensive 10-Year Strategy for Cancer Control and for Cancer Research from the Ministry of Health, Labor and Welfare of Japan, and in part by the National Cancer Center Research and Development Fund (23-A-9).

\section{References}

1. Yasui W. Future perspectives of gastric cancer treatment: from bench to bedside. Pathobiology. 2011;78:293-4.

2. Lauren P. The two histologcal main types of gastric carcinoma: diffuse and so-called intestinal-type carcinoma. An attempt at histo-clinical classification. Acta Pathol Microbiol Scand. 1965;64:31-49.

3. Nakamura K, Sugano H, Takagi K. Carcinoma of the stomach in incipient phase: its histogenesis and histological appearances. Gan. 1968;59:251-8.

4. Tatematsu M, Ichinose M, Miki K, Hasegawa R, Kato T, Ito N. Gastric and intestinal phenotypic expression of human stomach cancers as revealed by pepsinogen immunohistochemistry and mucin histochemistry. Acta Pathol Jpn. 1990;40:494-504.

5. Tajima Y, Yamazaki K, Nishino N, Morohara K, Yamazaki T, Kaetsu $\mathrm{T}$, et al. Gastric and intestinal phenotypic marker expression in gastric carcinomas and recurrence pattern after surgery-immunohistochemical analysis of 213 lesions. $\mathrm{Br} \mathrm{J}$ Cancer. 2004;91:1342-8.

6. Wakatsuki K, Yamada Y, Narikiyo M, Ueno M, Takayama T, Tamaki $\mathrm{H}$, et al. Clinicopathological and prognostic significance of mucin phenotype in gastric cancer. J Surg Oncol. 2008;98:124-9.

7. Lockhart DJ, Dong H, Byrne MC, Follettie MT, Gallo MV, Chee MS, et al. Expression monitoring by hybridization to high-density oligonucleotide arrays. Nat Biotechnol. 1996;14:1675-80.

8. Velculescu VE, Zhang L, Vogelstein B, Kinzler KW. Serial analysis of gene expression. Science. 1995;270:484-7.

9. Oue N, Hamai Y, Mitani Y, Matsumura S, Oshimo Y, Aung PP, et al. Gene expression profile of gastric carcinoma: identification of genes and tags potentially involved in invasion, metastasis, and carcinogenesis by serial analysis of gene expression. Cancer Res. 2004;64:2397-405.

10. Oue N, Mitani Y, Aung PP, Sakakura C, Takeshima Y, Kaneko $\mathrm{M}$, et al. Expression and localization of Reg IV in human neoplastic and non-neoplastic tissues: Reg IV expression is associated with intestinal and neuroendocrine differentiation in gastric adenocarcinoma. J Pathol. 2005;207:185-98.

11. Sentani K, Oue N, Tashiro T, Sakamoto N, Nishisaka T, Fukuhara $\mathrm{T}$, et al. Immunohistochemical staining of Reg IV and claudin-18 is useful in the diagnosis of gastrointestinal signet ring cell carcinoma. Am J Surg Pathol. 2008;32:1182-9.

12. Oue N, Sentani K, Noguchi T, Ohara S, Sakamoto N, Hayashi T, et al. Serum olfactomedin 4 (GW112, hGC-1) in combination with Reg IV is a highly sensitive biomarker for gastric cancer patients. Int J Cancer. 2009;125:2383-92.

13. Sentani K, Oue N, Sakamoto N, Arihiro K, Aoyagi K, Sasaki H, et al. Gene expression profiling with microarray and SAGE identifies PLUNC as a marker for hepatoid adenocarcinoma of the stomach. Mod Pathol. 2008;21:464-75.

14. Sentani K, Oue N, Sakamoto N, Anami K, Naito Y, Aoyagi K, et al. Upregulation of connexin 30 in intestinal phenotype gastric cancer and its reduction during tumor progression. Pathobiology. 2010;77:241-8.

15. Anami K, Oue N, Noguchi T, Sakamoto N, Sentani K, Hayashi T, et al. Search for transmembrane protein in gastric cancer by the Escherichia coli ampicillin secretion trap: expression of DSC2 in gastric cancer with intestinal phenotype. J Pathol. 2010;221:275-84.

16. Anami K, Oue N, Noguchi T, Sakamoto N, Sentani K, Hayashi T, et al. TSPAN8, identified by Escherichia coli ampicillin secretion trap, is associated with cell growth and invasion in gastric cancer. Gastric Cancer. doi:10.1007/s10120-015-0478-z.

17. Yamamoto T, Kato Y, Shibata N, Sawada T, Osawa M, Kobayashi M. A role of fukutin, a gene responsible for Fukuyama type congenital muscular dystrophy, in cancer cells: a possible role to suppress cell proliferation. Int J Exp Pathol. 2008;89:332-41.

18. Association Japanese Gastric Cancer. Japanese classification of gastric carcinoma: 3rd English edition. Gastric Cancer. 2011;14:101-12.

19. Kondo T, Oue N, Yoshida K, Mitani Y, Naka K, Nakayama H, et al. Expression of POT1 is associated with tumor stage and telomere length in gastric carcinoma. Cancer Res. 2004;64:523-9.

20. Yasui W, Ayhan A, Kitadai Y, Nishimura K, Yokozaki H, Ito H, et al. Increased expression of $\mathrm{p} 34 \mathrm{cdc} 2$ and its kinase activity in human gastric and colonic carcinomas. Int $\mathrm{J}$ Cancer. 1993;53:36-41.

21. Sentani K, Oue N, Naito Y, Sakamoto N, Anami K, Oo HZ, et al. Upregulation of HOXA10 in gastric cancer with the intestinal mucin phenotype: reduction during tumor progression and favorable prognosis. Carcinogenesis. 2012;33:1081-8.

22. Sekiguchi M, Sakakibara K, Fujii G. Establishment of cultured cell lines derived from a human gastric carcinoma. Jpn J Exp Med. 1978;48:61-8.

23. Yanagihara K, Seyama T, Tsumuraya M, Kamada N, Yokoro K. Establishment and characterization of human signet ring cell gastric carcinoma cell lines with amplification of the c-myc oncogene. Cancer Res. 1991;51:381-6.

24. Ochiai A, Yasui W, Tahara E. Growth-promoting effect of gastrin on human gastric carcinoma cell line TMK-1. Jpn J Cancer Res. 1985;76:1064-71.

25. Alley MC, Scudiero DA, Monks A, Hursey ML, Czerwinski MJ, Fine DL, et al. Feasibility of drug screening with panels of human tumor cell lines using a microculture tetrazolium assay. Cancer Res. 1988;48:589-601. 
26. The Cancer Genome Atlas Research Network. Comprehensive molecular characterization of gastric adenocarcinoma. Nature. 2014;513:202-9.

27. Shinmei S, Sentani K, Hayashi T, Sakamoto N, Goto K, Zarni Oo $\mathrm{H}$, et al. Identification of PRL1 as a novel diagnostic and therapeutic target for castration-resistant prostate cancer by the Escherichia coli ampicillin secretion trap (CAST) method. Urol Oncol. 2014;32:769-78.

28. Saito F, Matsumura K. Fukuyama-type congenital muscular dystrophy and defective glycosylation of $\alpha$-dystroglycan. Skelet Muscle. 2011;1:22.

29. Zhou Y, Jiang D, Thomason DB, Jarrett HW. Laminin-induced activation of Rac1 and JNKp46 is initiated by Src family kinases and mimics the effects of skeletal muscle contraction. Biochemistry. 2007;46:14907-16.

30. Kobayashi K, Nakahori Y, Miyake M, Matsumura K, Kondo-Iida E, Nomura Y, et al. An ancient retrotransposal insertion causes Fukuyama-type congenital muscular dystrophy. Nature. 1998;394:388-92.

31. Michele DE, Barresi R, Kanagawa M, Saito F, Cohn RD, Satz JS, et al. Post-translational disruption of dystroglycan-ligand interactions in congenital muscular dystrophies. Nature. 2002;418:417-22.

32. Perry JR, Stolk K, Franceschini N, Lunetta KL, Zhai G, McArdle $\mathrm{PF}$, et al. Meta-analyisis of genome-wide association data identifies two loci influencing age at menarche. Nat Genet. 2009;41:648-50.
33. Fleischmann A, Schlomm T, Huland H, Köllermann J, Simon P, Mirlacher M, et al. Distinct subcellular expression patterns of neutral endopeptidase (CD10) in prostate cancer predict diverging clinical courses in surgically treated patients. Clin Cancer Res. 2008;14:7838-42.

34. Kajiyama H, Shibata K, Terauchi M, Morita T, Ino K, Mizutani $\mathrm{S}$, et al. Neutral endopeptidase 24.11/CD10 suppresses progressive potential in ovarian carcinoma in vitro and in vivo. Clin Cancer Res. 2005;11:1798-808.

35. Luo Y, Fujii K, Ohmori H, Sasahira T, Moriwaka Y, Isobe M, et al. Antisense phosphorothioate oligodeoxynucleic acid for CD10 suppresses liver metastasis of colorectal cancer. Pathobiology. 2009;76:267-73.

36. Sato Y, Itoh F, Hinoda Y, Ohe Y, Nakagawa N, Ueda R, et al. Expression of CD10/neutral endopeptidase in normal and malignant tissues of the human stomach and colon. J Gastroenterol. 1996;31:12-7.

37. Huang WB, Zhou XJ, Chen JY, Zhang LH, Meng K, Ma HH, et al. CD10-positive stromal cells in gastric carcinoma: correlation with invasion and metastasis. Jpn J Clin Oncol. 2005;35:245-50.

38. Yamamoto T, Kato Y, Hiroi A, Shibata N, Osawa M, Kobayashi M. Post-transcriptional regulation of fukutin in an astrocytoma cell line. Int J Exp Pathol. 2012;93:46-55.

39. Kuang RG, Kuang Y, Luo QF, Zhou CJ, Ji R, Wang JW. Expression and significance of Musashi-1 in gastric cancer and precancerous lesions. World J Gastroenterol. 2013;19:6637-44. 